\title{
TWEAK induces liver progenitor cell proliferation
}

\author{
Aniela Jakubowski, ${ }^{1}$ Christine Ambrose, ${ }^{1}$ Michael Parr, ${ }^{1}$ John M. Lincecum, ${ }^{1}$ Monica Z. Wang, ${ }^{1}$ \\ Timothy S. Zheng, ${ }^{1}$ Beth Browning, ${ }^{1}$ Jennifer S. Michaelson, ${ }^{1}$ Manfred Baestcher, ${ }^{2}$ \\ Bruce Wang, ${ }^{3}$ D. Montgomery Bissell, ${ }^{3}$ and Linda C. Burkly ${ }^{1}$
}

\author{
1Departments of Exploratory Science, Discovery Biology, and Validation Biology, Biogen Idec Inc., Cambridge, Massachusetts, USA. \\ 2Department of Molecular and Medical Genetics, Oregon Health Sciences University, Portland, Oregon, USA. \\ 3Division of Gastroenterology and Liver Center, University of California San Francisco, San Francisco, California, USA.
}

\begin{abstract}
Progenitor ("oval") cell expansion accompanies many forms of liver injury, including alcohol toxicity and submassive parenchymal necrosis as well as experimental injury models featuring blocked hepatocyte replication. Oval cells can potentially become either hepatocytes or biliary epithelial cells and may be critical to liver regeneration, particularly when hepatocyte replication is impaired. The regulation of oval cell proliferation is incompletely understood. Herein we present evidence that a TNF family member called TWEAK (TNF-like weak inducer of apoptosis) stimulates oval cell proliferation in mouse liver through its receptor Fn14. TWEAK has no effect on mature hepatocytes and thus appears to be selective for oval cells. Transgenic mice overexpressing TWEAK in hepatocytes exhibit periportal oval cell hyperplasia. A similar phenotype was obtained in adult wild-type mice, but not Fn14-null mice, by administering TWEAK-expressing adenovirus. Oval cell expansion induced by 3,5-diethoxycarbonyl-1,4-dihydrocollidine (DDC) was significantly reduced in Fn14-null mice as well as in adult wild-type mice with a blocking anti-TWEAK mAb. Importantly, TWEAK stimulated the proliferation of an oval cell culture model. Finally, we show increased Fn14 expression in chronic hepatitis $\mathrm{C}$ and other human liver diseases relative to its expression in normal liver, which suggests a role for the TWEAK/Fn14 pathway in human liver injury. We conclude that TWEAK has a selective mitogenic effect for liver oval cells that distinguishes it from other previously described growth factors.
\end{abstract}

\section{Introduction}

Compensatory epithelial hyperplasia following liver injury involves 1 of 3 different cellular compartments, depending on the nature of the injury. With simple loss of hepatocyte mass, the paradigm of which is partial hepatic resection, hepatocytes within the remaining lobes proliferate. When the injury consists of biliary obstruction, proliferation of the biliary epithelium is prominent. Injury that both reduces hepatocyte mass and impairs hepatocyte proliferation elicits the appearance of "oval" cells. Oval cells arise from the portal area of the lobule, which is the location of the putative hepatic stem cell. These cells are viewed as a progenitor cell type because of their known ability to differentiate into either hepatocytes or biliary epithelial cells (BECs) (1). With the development of improved techniques for detecting oval cells in liver sections, it has become apparent that the oval cell compartment participates in a range of injuries to human liver (2) and may be critical to the survival of patients with acute liver failure $(3,4)$. There are many rodent models for oval cell proliferation. A commonly used model involves treating rats with the carcinogen 2-acetylaminofluorene (2-AAF) followed by a growth stimulus in the form of partial hepatectomy $(\mathrm{AAF} / \mathrm{PH})$ or carbon

Nonstandard abbreviations used: AAF, acetylaminofluorene; AAT, $\alpha 1$ antitrypsin; ALT, alanine aminotransferase; BEC, biliary epithelial cell; DDC, 3,5-diethoxycarbonyl-1,4-dihydrocollidine; HCC, hepatocellular carcinoma; NASH, nonalcoholic steatohepatitis; NRC-1, normal rat cholangiocyte-1; Ntg, nontransgenic; PCNA, proliferating cell nuclear antigen; $\mathrm{PH}$, partial hepatectomy; TWEAK, TNF-like weak inducer of apoptosis.

Conflict of interest: The authors have declared that no conflict of interest exists.

Citation for this article: J. Clin. Invest. 115:2330-2340 (2005).

doi:10.1172/JCI23486. tetrachloride (AAF/CCl4). This results in impressive growth of a population of small ovoid cells with a large ratio of nucleus to cytoplasm (5-7). These oval cells express phenotypic markers of both immature hepatocytes and BECs, such as $\alpha$-fetoprotein and cytokeratins 8 and 18 respectively (7-9).

Hepatic regeneration after $\mathrm{PH}$ is a multistep process involving numerous cytokines and growth factors. HGF, EGF, and TGF- $\alpha$ are viewed as primary stimuli but require that hepatocytes be primed by other soluble factors, including TNF and IL-6 $(1,10)$. Some of the same factors have been implicated in oval cell proliferation $(1,9,11-15)$. However, their effects are not specific for oval cells, which raises the question of whether they are both necessary and sufficient.

TNF-like weak inducer of apoptosis (TWEAK) (16) is a member of the TNF- $\alpha$ family of growth regulators that has weak apoptotic activity for certain human tumor cell lines (17). Its mRNA is broadly expressed in normal tissues, including the liver. Studies using an array of cell types have indicated that its putative actions are broad and include regulation of cell survival, proliferation and migration, and induction of proinflammatory cytokines and chemokines (18). A TWEAK receptor known as Fn14, a member of the TNF receptor family, has been identified $(19,20)$. Its expression is restricted to epithelial and mesenchymal cell types. In the liver of the growing newborn, Fn14 is highly expressed (21). It is minimally expressed in the adult except during injury and repair, such as following $\mathrm{PH}$ in mice and hepatocellular carcinoma (HCC) in mice and humans (20). In the course of studies exploring the biological role of TWEAK, we prepared a transgenic mouse expressing the gene under control of the $\alpha 1$ antitrypsin (AAT) promoter, which is primarily expressed in the liver (22). The livers in these mice exhibited a striking periportal 

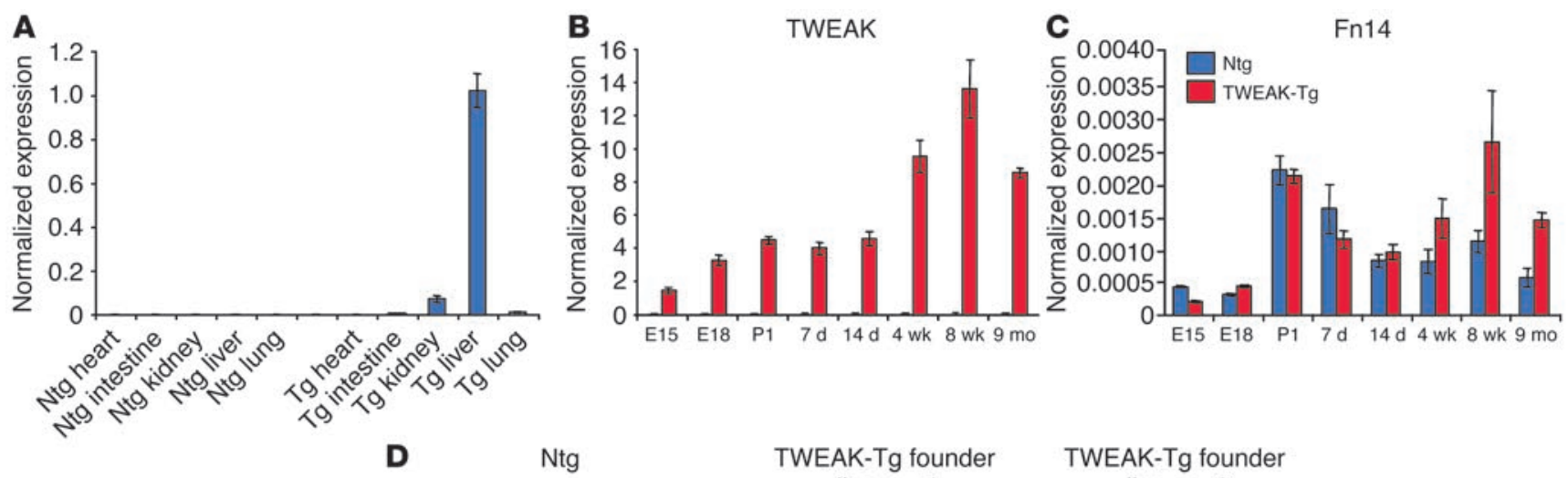

Ntg

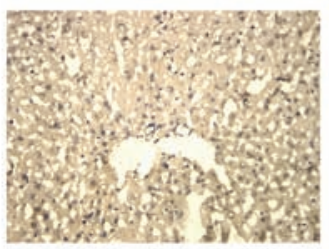

TWEAK-Tg founder line no. 1

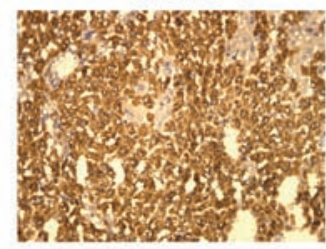

TWEAK-Tg founder line no. 2

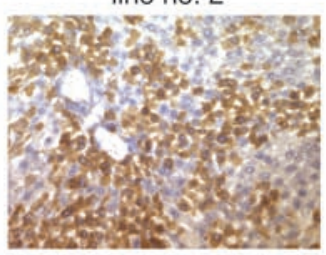

Figure 1

TWEAK transgene is expressed primarily in the livers of 2 independent transgenic lines. (A) AAT promoter directs expression of TWEAK transgene to the liver and at low levels to the kidney. Quantitative PCR using transgene-specific primers was done with cDNA pooled from 3 adult transgenic animals. Data was normalized to the levels of the GAPDH gene. (B) Time course of TWEAK and (C) Fn14 mRNA expression in TWEAK-Tg vs. Ntg mice was measured by quantitative PCR. Each time point represents the combined RNA from 3 different animals. (D) TWEAK protein expression in 2 independent transgenic founder lines as compared with that in a control littermate. TWEAK protein was detected by staining frozen liver sections with a hamster anti-TWEAK mAb.

outgrowth of oval cells but otherwise appeared healthy. The findings were confirmed by transiently overexpressing TWEAK in the adult mouse liver and by chemically inducing oval cell proliferation in the mouse. In the latter model, anti-TWEAK mAb blocked oval cell expansion. TWEAK overexpression in these models had no detectable effect on hepatocyte proliferation. Moreover, the TWEAK receptor, Fn14, was present on ductal structures and oval cells. In addition, TWEAK was shown to be mitogenic in an oval cell culture model. We concluded that TWEAK initiates proliferation of oval cells and is specific to this population. The potential relevance to human liver disease is supported by additional data demonstrating increased Fn14 expression on bile ducts and ductlike structures in human cirrhotic liver.

\section{Results}

TWEAK transgenic mice. Transgenic mice carrying full-length murine TWEAK cDNA under the murine AAT promoter (TWEAK-Tg) were prepared. Two lines were developed. TWEAK transgene expression was predominantly hepatic, but low levels were also detected in the kidney by quantitative PCR (Figure 1A). The developmental expression of TWEAK and Fn 14 was also analyzed by quantitative PCR. In nontransgenic (Ntg) mice, TWEAK expression was very low at all tested times. In contrast, in TWEAK-Tg mice, TWEAK was detectable at low levels as early as E15.5, and its expression continuously increased, reaching high levels in the adult (Figure 1B). In both TWEAK-Tg and Ntg animals, Fn14 expression only reached significant levels beginning at birth: it remained relatively high in adult and older transgenic mice but decreased in the age-matched Ntg mice, which suggests that it is subject to ligand regulation (Figure 1C).
One founder line strongly expressed TWEAK protein in the liver in hepatocytes and focally and weakly in the kidney (Figure 1D); the protein was not detected in other organs. The second founder line exhibited a weaker expression of TWEAK protein in the liver. Circulating TWEAK protein at levels of $8-25 \mathrm{ng} / \mathrm{ml}$ was detected only in the high-expressing line.

Transgenic TWEAK overexpression results in oval cell proliferation. An increased number of oval cells, defined as small cells with an oval nucleus and scant cytoplasm, spreading from the periportal region was observed in TWEAK-Tg mice as compared with Ntg mice. This phenotype was observed as early as 2 weeks of age (data not shown). By 8 weeks and thereafter to 5-8 months, TWEAK-Tg livers exhibited a relatively high number of cells organized either in single file or in duct-like structures in the periportal region and extending into the liver lobule. (Figure 2, C and D). The majority of the ductlike structures were irregularly shaped with a poorly defined lumen. The livers of Ntg littermates were normal, each portal tract having 1 or 2 well-formed bile ducts (Figure 2, A and B).

We used immunohistochemical staining of serial sections to further characterize the proliferating cells. As in previously published work, A6 antigen $(23,24)$ and the hematopoietic marker CD34 (25) were expressed by both proliferating oval cells and normal BECs (26). In control mice, A6 and CD34 coexpression was detectable only in bile ducts (Figure 2, E-G), with CD34 being present also in the portal vein endothelium (data not shown). In contrast, TWEAK-Tg livers exhibited an increased number of periportal A6and CD34-expressing cells (Figure 2, H and I), with some cells displaying both markers (Figure 2J). The data indicate that TWEAK-Tg mice have increased numbers of oval cells based on their morphology, location, and marker expression. The immaturity of the cells 
A

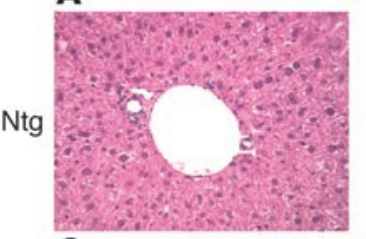

C

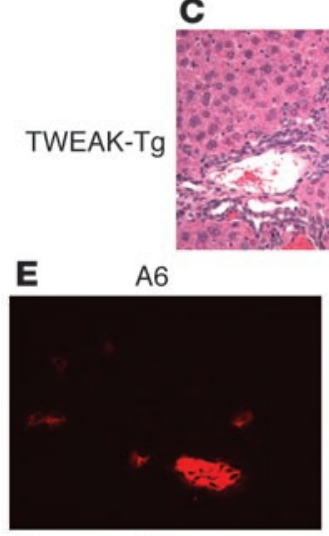

H

A6

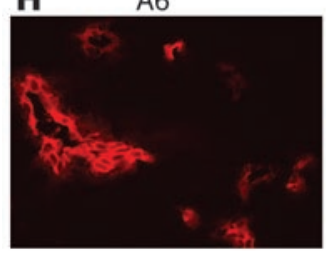

Figure 2

TWEAK overexpression promotes oval cell hyperplasia. (A-D) H\&E staining of 8to 10 -week-old (A and $\mathbf{B}) \mathrm{Ntg}$ or (C and D) TWEAK-Tg livers. Magnification, $\times 320$ (A and C); ×500 (B and D). (E-G) A6 (red) and CD34 (green) expression in Ntg livers. (E) A6 expression on bile duct epithelium. (F) CD34 expression on bile duct epithelium. (G) Coexpression of A6 and CD34. (H-J) TWEAK-Tg livers showing increased expression of (H) A6 and (I) CD34 in oval cells and bile ducts and (J) increased coexpression of A6 and CD34. Original magnification, $\times 200(\mathbf{E}-\mathbf{J})$.
TWEAK-Tg mice had normal serum transaminases and no detectable inflammation or bile deposits (data not shown). Taken together, the data indicate that the proliferative phenotype is not due to an impaired hepatocyte response to injury.

Fn14 expression is associated with BECs and periportal cells. If oval cells respond directly to TWEAK, they presumably express the TWEAK receptor, Fn14. This was examined by in situ hybridization with both embryo and adult livers. At E18.5 both transgenic (Figure 4E) and control mice (Figure 4A) expressed similar levels of Fn14 mRNA in the ductal plate cell layers surrounding the portal mesenchyme from which bile ductules emerge (27). Low levels of Fn14 were also uniformly expressed in the parenchyma. Fn14 expression peaked in newborn mice, being slightly increased in TWEAK-Tg mice (Figure 4F) relative to Ntg littermates (Figure 4B) and was also expressed in BCEs and surrounding periportal cells (Figure 4, B and F), with scattered and generally lower expression in the parenchyma. In the adult livers of both transgenic and control mice, Fn14 expression was sharply diminished. In Ntg adult mice, it was detectable mostly in BECs, in a few periportal cells, and at a very low level in the parenchyma (Figure 4, C and D). In contrast, in adult TWEAK-Tg liver, Fn14 expression was strikingly elevated in the region of oval cell hyperplasia (Figure 3, G and $\mathrm{H}$ ).

Role of TWEAK in adult mice. As shown in Figure 1B, TWEAK transgene mRNA expression is driven by the AAT promoter, is detectable as early as day 15 of fetal development, and, as previously reported, increases rapidly through day 18 (28). This raises the possibility that TWEAK exerts its effects mainly in utero rather than in the adult. This was explored in 2 different models. First we transiently overexpressed TWEAK in normal adult mouse liver using an adenoviral vector (Ad-TWEAK). Control mice received an equivalent number of viral particles containing GFP and lacking TWEAK cDNA is inferred from the fact that most are not organized as ductular structures; however, the latter were also present, suggesting that a range of maturation states may be present.

TWEAK is proapoptotic in some settings, which raises the possibility that the observed proliferation of oval cells was secondary to hepatocyte death. To test this, we examined proliferation and apoptosis in TWEAK-Tg relative to Ntg livers. Only periportal oval cells and BECs exhibited significantly increased proliferation in TWEAK-Tg relative to that in Ntg mice (Figure 3A) while hepatocyte proliferation was similar in both (Figure 3B). The increased proliferation of oval cells in the TWEAK-Tg mice was evident as early as 2 weeks of age and persisted thereafter although it decreased somewhat with age. Cell death was examined with TUNEL staining (Figure 3, C and D). At 2 weeks and 8 weeks, when oval cell proliferation was most evident, there was no difference in hepatocyte apoptosis between the TWEAK-Tg and Ntg mice (Figure 3D). At 7 months, the TWEAK-Tg liver showed an increase in apoptotic hepatocytes relative to a very low rate of apoptosis in age-matched controls. The significance of this finding is uncertain. Interestingly, at early time points, oval cell apoptosis was increased (Figure 3C), suggesting that increased proliferation in TWEAK-Tg mice is accompanied by a detectable increase in cell turnover. Finally,
(Ad-control), and transduction was confirmed by GFP expression in the liver (data not shown). Seven days after Ad-TWEAK administration, the liver exhibited a remarkable proliferation of oval cells, some of which were organized in duct-like structures (Figure 5A). The identity of the cells was determined both by morphological criteria and by the coexpression of A6 and CD34 markers (Figure 5, $\mathrm{B}$ and $\mathrm{C})$. Mice receiving the control adenovirus showed a minimal amount of ductal proliferation (Figure 5, D-F).

Adenoviruses caused a mild inflammatory response and limited hepatocyte damage that peaked by day 7 . Since TWEAK was reported to induce apoptosis in some systems (29-32), we wished to rule out the possibility that TWEAK and an adenoviral vector could act synergistically to enhance hepatocyte damage and thus stimulate oval cell proliferation. Therefore, we assessed serum alanine aminotransferase (ALT) levels and apoptosis in the liver both prior to and at the peak of the inflammatory response. We found normal ALT values and very few apoptotic hepatocytes in both Ad-TWEAK- and Ad-control-treated mice prior to day 7 (Figure 5, G and H).

The second model we used to test the role of TWEAK in adult mice was a liver injury model in which oval cell proliferation was induced chemically by 3,5-diethoxycarbonyl-1,4-dihydrocollidine (DDC) feeding. First we asked whether the TWEAK pathway is 
A

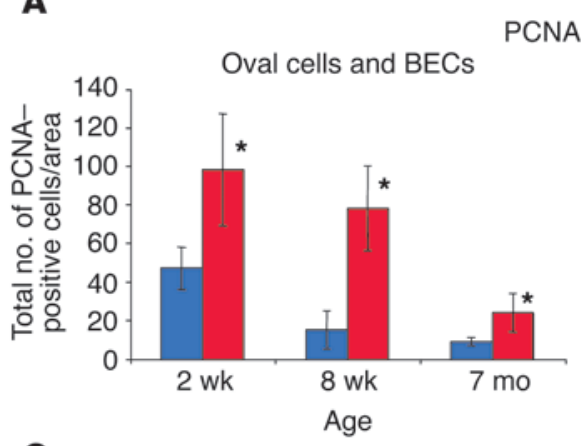

C

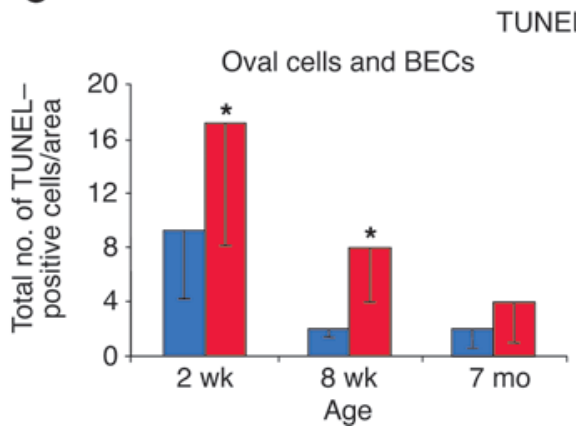

B

D
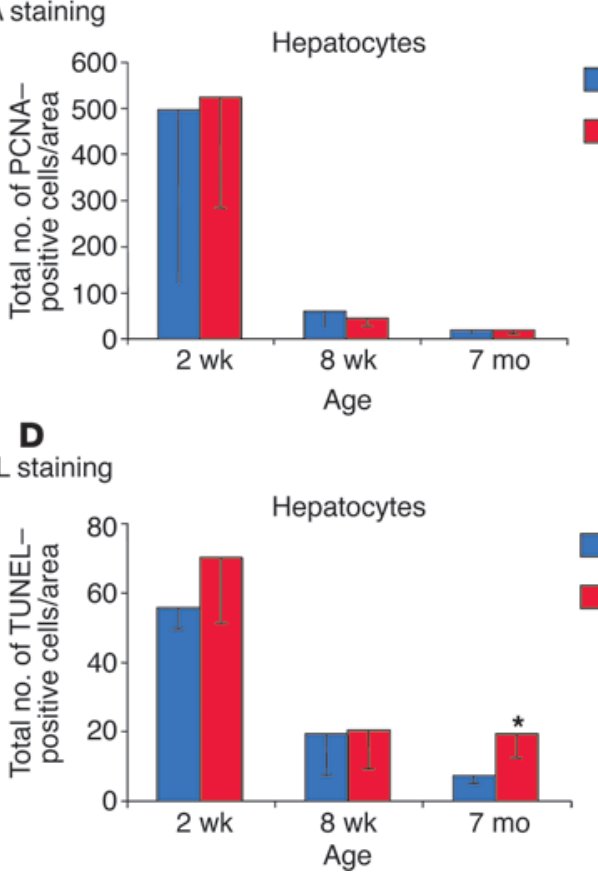

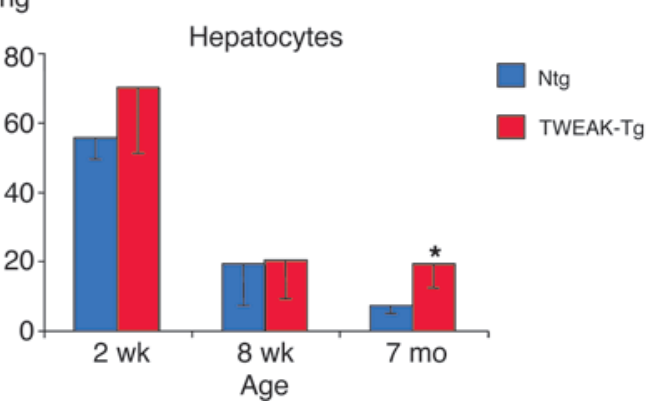

\section{Figure 3}

TWEAK overexpression in transgenic mice promotes early proliferation and increased turnover of oval cells with no detectable damage in the parenchyma. (A and B) Proliferation, as measured by PCNA staining of TWEAK-Tg and Ntg livers at the indicated ages. (A) PCNA-positive periportal oval cells and BECs show increased proliferation in TWEAK-Tg vs. Ntg livers. (B) PCNA-positive hepatocytes. (C and D) Apoptosis detected by TUNEL staining of TWEAK-Tg and Ntg livers at the indicated ages. (C) Apoptotic periportal oval cells and BECs show increased proliferation in TWEAK-Tg vs. Ntg livers. (D) Apoptotic hepatocytes. PCNAor TUNEL-positive cells were counted in 20 different fields (magnification, $\times 200$ ). ${ }^{*} P<0.05$ by 2 -tailed Student's $t$ test. expressed in this model. Seven days after the start of DDC feeding, we found that Fn14 expression was markedly upregulated in the reactive portal area (Figure 6B) in contrast to Fn14 expression in control mice (Figure 6A), supporting the possibility that TWEAK pathways play a role in the regulation of these cells. The scattered and low expression of Fn14 in the lobule was similar in DDCtreated and control mice. Expression of TWEAK itself in control mice was present across the lobule, possibly in resident stellate and Kupffer cells (Figure 6C). In comparison, in DDC-fed mice, TWEAK was strikingly elevated in reactive portal areas; it appeared also to be slightly higher in the parenchyma (Figure 6D). When DDC-fed mice were treated with a blocking anti-TWEAK mAb, the proliferation of A6-positive oval cells was significantly inhibited (Figure 7, D and E) relative to that in untreated DDC-fed mice and control IgG-treated DDC-fed mice (Figure 7, B and C). Control Ig had no effect on the DDC-induced progenitor cell proliferation
Figure 4

Increased Fn14 mRNA expression in the hyperplastic regions of adult TWEAK-Tg relative to Ntg livers. Fn14 mRNA expression was detected by in situ hybridization using a murine Fn14 riboprobe template. (A-D) Ntg liver in (A) E18.5 embryo, (B) newborn, and (C) 8-weekold mouse. Darkfield (top) and brightfield (bottom) views are shown. Magnification, $\times 250$. (D) Ntg 8-week-old liver. Magnification, $\times 640 .(E-H)$ TWEAK-Tg liver in (E) E18.5 embryo, (F) newborn, and (G) 8-week-old mouse. Darkfield (top) and brightfield (bottom) views are shown. Magnification, $\times 250$. (H) TWEAK-Tg 8-week-old liver. Magnification, $\times 640$.
A
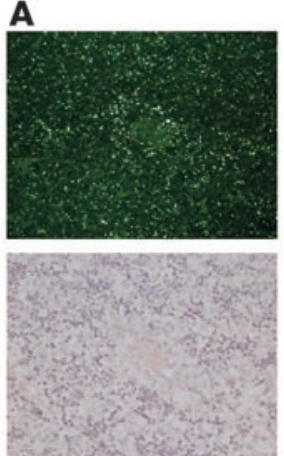

E

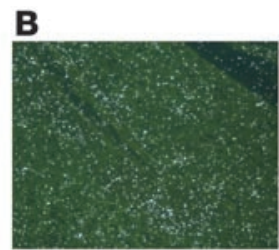

C

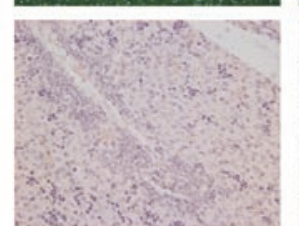

$\mathbf{F}$
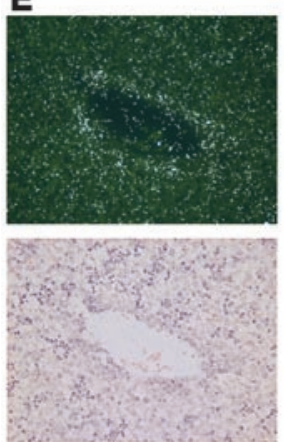

G

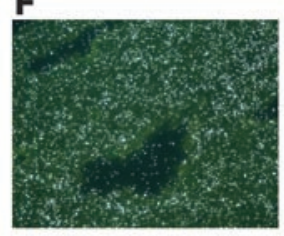

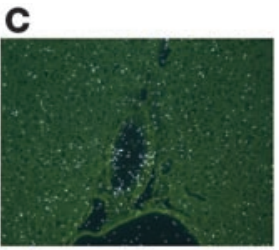

D
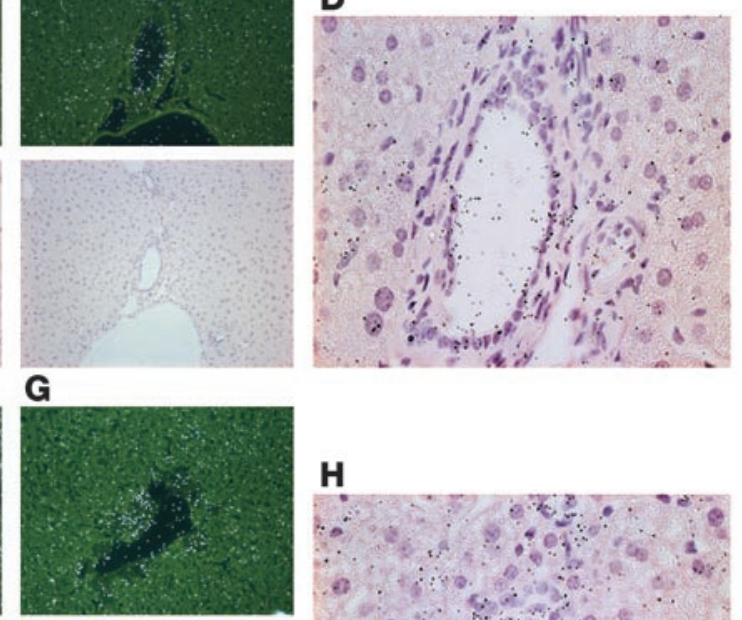

H

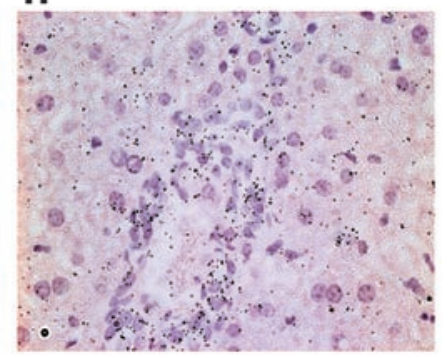


A

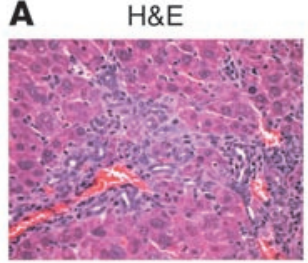

Ad-TWEAK

D

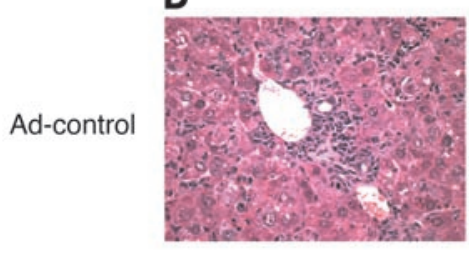

G

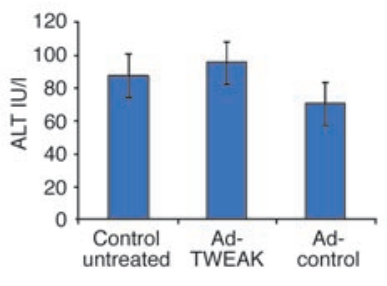

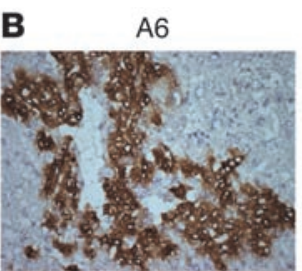

E

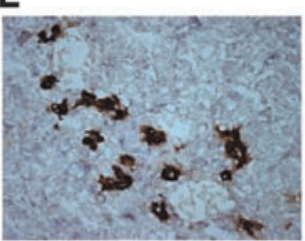

H

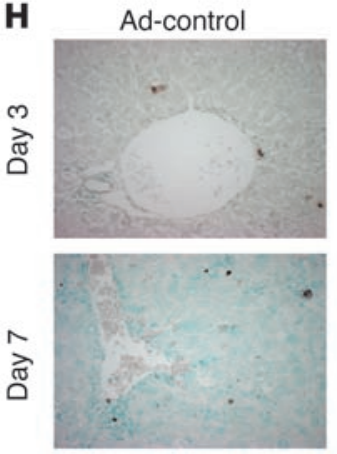

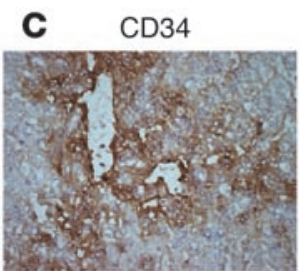

$\mathbf{F}$

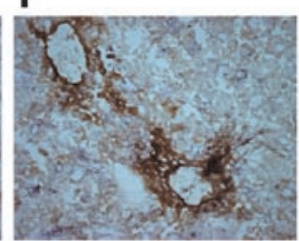

Ad-TWEAK

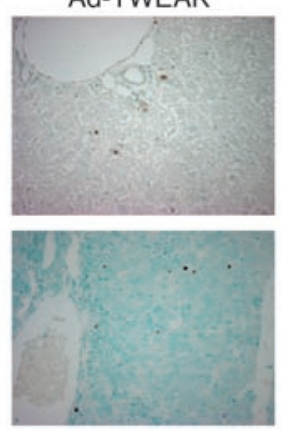

Figure 5

Ad-TWEAK administration in adult mice induces oval cell and biliary duct hyperplasia in the absence of significant liver damage. Mice were dosed with either Ad-TWEAK or Ad-control, as described in Methods, and livers were obtained 3 or 7 days later for TUNEL staining or 7 days later for $\mathrm{H} \& E, A 6$, and CD34 staining. Serial frozen sections were stained with A6 or CD34 in order to assess marker coexpression. (A-C) Ad-TWEAK treated mice. (A) H\&E staining showing a marked oval cell and ductal hyperplasia. (B) Extensive A6 and (C) CD34 coexpression on oval cells and duct-like cells and on BECs. (D-F) Ad-controltreated mice. (D) H\&E staining showing an inflammatory response induced by Ad-control vector. (E) A6 and (F) CD34 coexpressed on bile ducts in the portal tract region and CD34 additionally expressed on vessels. Magnification, $\times 200$. (G) Serum ALT measured in IU/I 3 days after adenoviral administration, showing no signs of liver damage. $(\mathbf{H})$ TUNEL staining at days 3 and 7 after Ad-control or Ad-TWEAK administration. Magnification, $\times 400$.

compared with the untreated DDC-fed mice (Figure 7, B and C). We conclude from these 2 models that TWEAK causes proliferation with selectivity for oval cells in adult mice.

TWEAK-induced oval cell proliferation is Fn14 dependent. Accumulating evidence suggests that Fn14 is the receptor for TWEAK, although some studies have opened the possibility of additional receptors (18). In order to examine the role of Fn14 in mediating the mitogenic effect of TWEAK, we recently generated Fn14-null mice (Fn14-KO), which were fertile and viable with no gross or histological abnormality. For this purpose, the 2 animal models described above were utilized in the Fn14-KO mice. As described above and shown by A6 staining in Figure 5B, Ad-TWEAK caused a prominent oval cell and ductal hyperplasia in the wild-type mice (Figure 8A) relative to Ad-control-treated wild-type mice, which showed no significant proliferation (Figure 8C). However, Fn14-KO mice administered Ad-TWEAK exhibited no hyperplastic phenotype (Figure 8B), and their livers appeared histologically similar to those of Ad-control-treated wild-type or Fn14-KO mice (Figure 8, C-E), indicating that the mitogenic effect of TWEAK on oval cells in the liver is exclu- sively Fn14 dependent. Thus, the numbers of A6positive cells in the Fn14-KO mice treated with Ad-TWEAK were low and comparable to those in either wild-type or Fn14-KO mice treated with Ad-control, as quantified by MetaMorph software (Figure 9E). We wished to test whether Fn14 also mediates oval cell proliferation in the physiological model of DDC feeding. As described above, DDC-fed wild-type mice exhibited marked oval cell proliferation (Figure 9B) relative to wild-type mice fed a regular diet (Figure 9A). DDC-fed Fn14-KO mice exhibited $40 \%$ less oval cell proliferation than DDC-fed wild-type mice (Figure 9, D and E). This reduction in oval cell proliferation was similar to the effect obtained with pharmacological blocking. There was no significant difference between the wild-type and Fn14-KO mice maintained on a regular diet, either histologically or in the amount of A6 expression (Figure 9, A and C). These data provide independent evidence for the role of the TWEAK/Fn14 pathway in the induction of oval cell proliferation.

TWEAK is mitogenic for normal rat cholangiocyte-1 cells. As a direct test of the mitogenic effect of TWEAK on bile duct-derived epithelial cells, this factor was added to the medium of normal rat cholangiocyte-1 (NRC-1) cells. The NRC-1 cell line expresses $\beta_{6}$ integrin and thus represents reactive BECs or mature oval cells (data not shown). The cultures were placed in serumfree medium for 24 hours, at which point they were growth-arrested. TWEAK was added and the response assessed by incorporation of $\mathrm{BrdU}$ into the nucleus. TWEAK stimulated DNA synthesis in a dose-dependent fashion. At the optimal concentration $(100 \mathrm{ng} / \mathrm{ml}), 40 \%$ of the culture was BrdU-positive (Figure 10).

Fn14 is expressed in BECs and duct-like structures in buman liver disease. Extending the results in mice to human liver disease, we asked whether Fn14 was expressed in diseases involving progenitor cell proliferation, such as chronic viral hepatitis, alcoholic liver disease, and nonalcoholic fatty liver disease $(3,33)$. In normal liver, we found that Fn14 is expressed in bile ducts and in some smooth muscle cells surrounding them (Figure 11A). Interestingly, increased Fn14 expression was detected in nonalcoholic steatohepatitis (NASH), alcoholic liver disease, and HCV with HCC (Figure 11, B-D), in which Fn14 was found in the abundant duct-like structures and in bile ducts. In some sections, Fn14 also appeared to be expressed in the fibrotic regions, possibly representing trapped ductal structures and smooth muscle cells.

\section{Discussion}

This report shows that TWEAK selectively stimulates proliferation of a hepatic progenitor cell population known as oval cells both when overexpressed in transgenic mice and when transiently expressed in adult mice. This mitogenic effect of TWEAK is mediated exclusively through Fn14. In addition, the physiological role of TWEAK as a novel stimulator of oval cell proliferation is demon- 


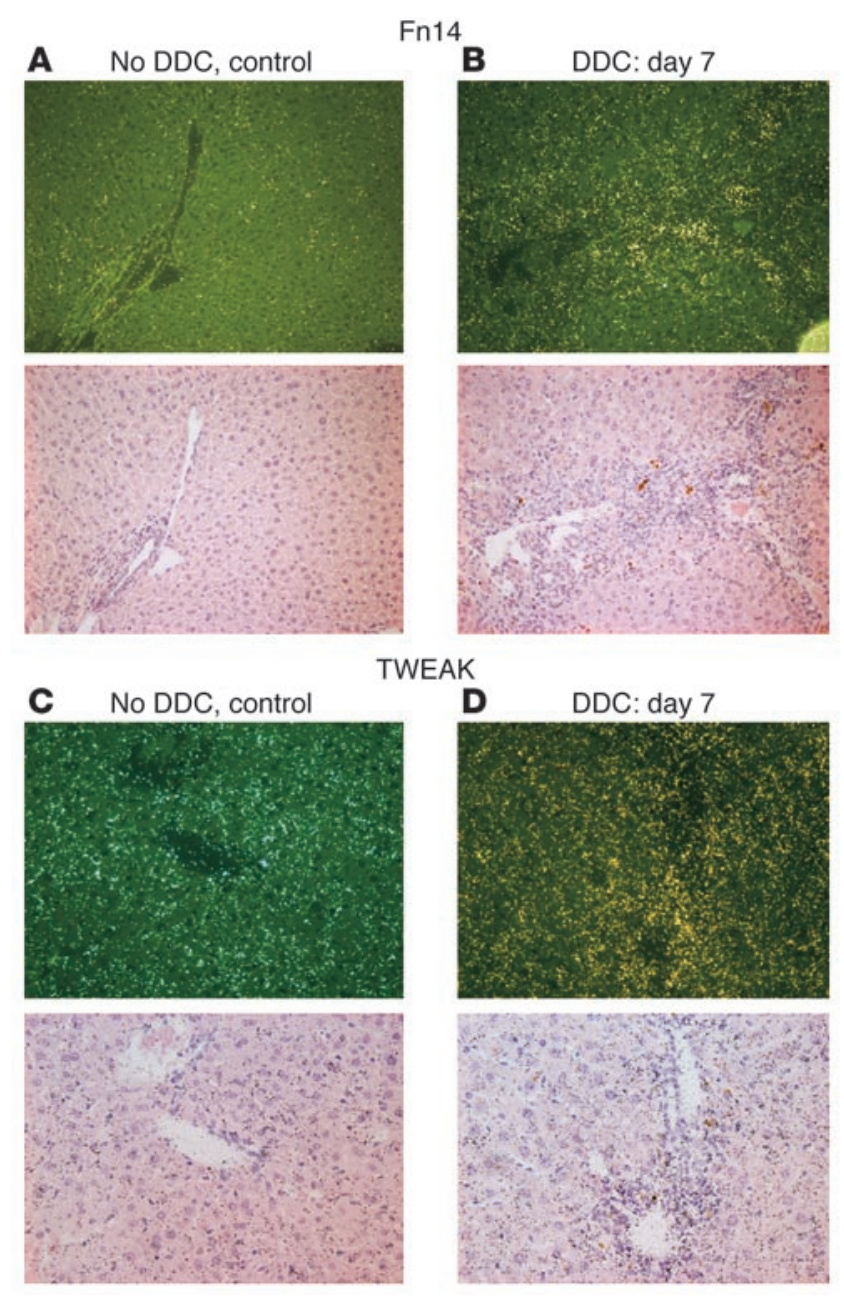

\section{Figure 6}

Fn14 and TWEAK are upregulated in the reactive portal area (including oval cells and bile ducts) in the DDC-fed mice but not in control mice fed regular diet with no DDC. Fn14 expression was detected by in situ hybridization. Dark- and brightfield pictures of the same field are displayed in (A) a control mouse on regular diet and (B) a DDC-fed mice on day 7. TWEAK expression in (C) control mouse on regular diet and (D) DDC-fed mouse on day 7. Magnification, $\times 250$.

strated in the DDC liver injury model, which features progenitor cell expansion. Since existing models of oval cell proliferation appear to require blockade of hepatocellular proliferation, we examined TWEAK-Tg mice for the presence of this condition and found no evidence for hepatic damage. In addition, we found that TWEAK pathway blockade had no effect on the regenerative response to $\mathrm{PH}$ (A. Jakubowski and D.M. Bissell, unpublished observations). Previous studies have provided evidence that HGF, TGF- $\alpha$, EGF, FGF, TNF- $\alpha$, IL- 6 , and leukemia-inhibitory growth factor (LIF) modulate oval cell proliferation in the context of injury $(34)(12,15,35-37)$. However, these growth factors also modulate hepatocyte proliferation in response to injury $(10,38,39)$. In contrast, TWEAK appears to be selective for oval cells, with no detectable mitogenic effect on hepatocytes, and represents the first such factor described.

The hyperplastic phenotype observed in the livers of both TWEAK-Tg mice and Ad-TWEAK-treated mice resembles that found in mice fed with choline-deficient, ethionine-containing
(CDE) diet as well as in the AAF/PH model of oval cell proliferation $(7,8,40)$. In the CDE model, individual OV-6- and $\alpha$-fetoprotein-positive periportal oval cells appear early, then differentiate into duct-like structures and later into hepatocytes (40). Similarly, individual unorganized oval cells were observed in 2-weekold TWEAK-Tg mice; these preceded the appearance of duct-like structures, found in adult 8-week-old mice, which suggests that these cells represent a less mature differentiation stage. As previously reported, progenitor cells expressed both A6 and CD34 markers (25). Some progenitor cells expressed CD34 but not A6, which suggests that they represent a less mature population $(26,41)$. Thus, it is likely that the observed oval cells represent various maturation stages, up to definitive BECs. However, A6 and CD34 markers are also expressed on mature BECs, and therefore we cannot rule out the possibility that some of the proliferating cells were BECs. Whether or not the TWEAK-elicited cells mature into hepatocytes, as do carcinogen-induced oval cells $(7,42)$, will be the focus of a future study. It will be important to determine whether TWEAK acts solely to expand the progenitor population or is also a differentiating factor for the biliary pathway. Interestingly, we observed no progression of the hyperplastic phenotype leading to oval cell accumulation in older animals nor increased liver mass. Instead, oval and ductal cells, but not hepatocytes, had a higher turnover in TWEAK-Tg mice compared with control mice, suggesting that TWEAK may contribute mainly to initiating progenitor cell expansion.

Fn14 is expressed on the ductal plate in the embryonic liver and on the biliary duct epithelium and a few periductal cells in the adult normal liver; such cells may include the hepatic stem cells that presumably reside in or near the canals of Hering. These findings further support the conclusion that TWEAK directly delivers growth signals to progenitor cells and BECs in vivo. It has been reported that hepatic stellate cells proliferate in close proximity to oval cells, elaborating growth factors that are mitogenic for oval cells (43). The participation of stellate cells in the induction of the hyperplastic phenotype cannot be excluded since they may also express Fn14 (D.M. Bissell, unpublished observations). However, this is less likely since the number of periportal cells with smooth muscle actin was unchanged in TWEAK-Tg mice, indicating an absence of activated stellate cells (data not shown). The role of TWEAK in driving oval cell proliferation was demonstrated also in the DDC chemical injury model for oval cell expansion, in which blocking anti-TWEAK $\mathrm{mAb}$ resulted in $35 \%$ inhibition of oval cell proliferation. A similar level of inhibition was also obtained in the DDC-fed Fn14-KO mice, thus supporting the conclusions obtained with the pharmacological inhibition: that the TWEAK/Fn14 pathway plays a role in stimulating oval cell proliferation. Since TWEAK blockade as well as the Fn14 deficiency did not result in complete inhibition of oval cell proliferation, other pathways appear to be involved in this function. We found that Fn14 mRNA was upregulated in oval cells and BECs in the reactive portal area of DDC-fed mice. The source of endogenous TWEAK in this model is speculative, but most likely the source is Kupffer cells or infiltrating macrophages, as suggested by TWEAK mRNA expression in monocyte/macrophage cell populations $(17,18)$. Although Fn14 was also weakly expressed on scattered hepatocytes, we found no evidence that TWEAK produced in the portal zone has any effect on lobular hepatocytes.

Recently, Polek et al. (44) examined the differentiating effect of TWEAK on a murine monocyte/macrophage cell line that does not display Fn14. Under the influence of TWEAK, the cells 

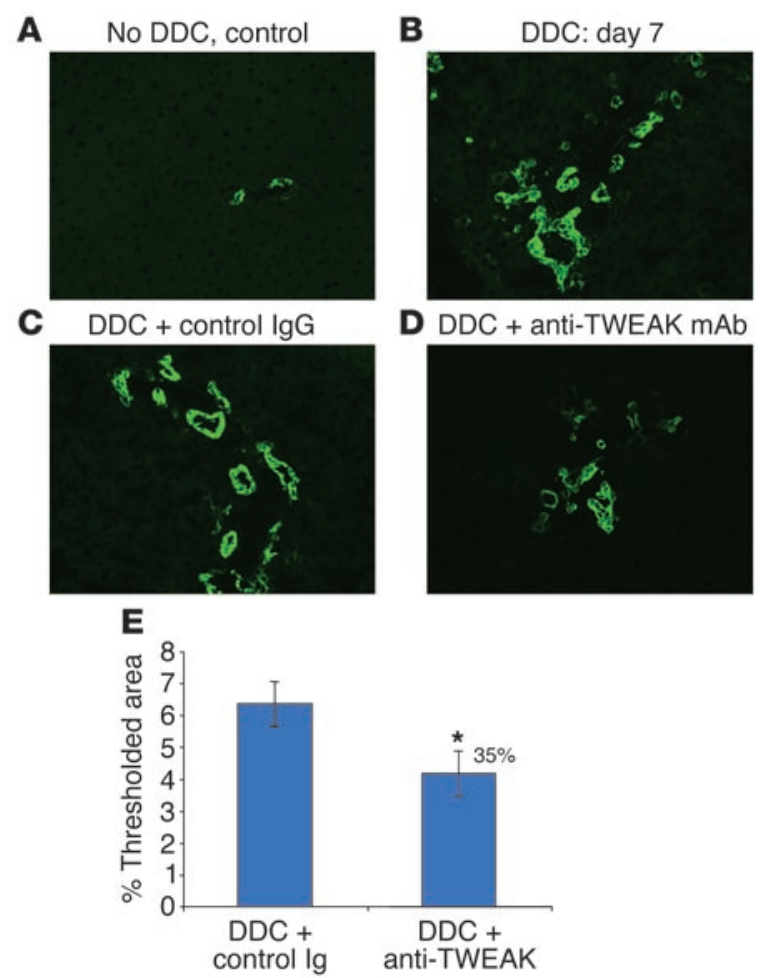

acquired the features of osteoclasts, which suggests the possibility of an additional TWEAK receptor. The nature of this putative receptor and its tissue distribution remain to be determined. In the liver, the mitogenic effect of TWEAK appears to be mediated solely by the known TWEAK receptor, Fn 14 .

The classical models of oval cell proliferation involve administration of carcinogens $(7,42)$. In general, these compounds block hepatocyte proliferation, and studies have shown that neoplasia in these models does not arise by dedifferentiation of mature hepatocytes. Thus, it is thought that cancer arises out of transformed oval cells $(3,7,42,45,46)$. Also, oval cells have been identified in human liver diseases associated with a high incidence of HCC or cholangiocarcinoma (such as chronic viral and alcoholic hepatitis, NASH, and sclerosing cholangitis) $(43,47,48)$. Our studies show that expression of the TWEAK receptor, Fn14, increases in some of these same diseases. On the other hand, we monitored our TWEAK-Tg mice for almost 1 year and found no indications of uncontrolled cell growth or frank neoplasia. Rather, the oval cell population reaches a plateau in which hyperplasia appears to be balanced by apoptosis.

In summary, our study reveals a novel role for TWEAK: initiation of oval cell proliferation, most likely by directly signaling to these Fn14 receptor-bearing cells. Unlike other growth factors

\section{Figure 8}

Mitogenic effect of TWEAK on oval cells is Fn14 dependent. Fn14-KO or wild-type mice were dosed with Ad-TWEAK or Ad-control as described in Methods, and frozen livers were obtained 7 days later and stained for $A 6$ expression. The amount of positive staining was then quantitated using MetaMorph software. Ad-TWEAK-treated (A) wild-type mice and (B) Fn14-KO mice. Ad-control-treated (C) wild-type mice and (D) Fn14-KO mice. Magnification, $\times 100$. (E) Quantitation of A6 staining using MetaMorph software. ${ }^{*} P<0.02$ by 2 -tailed Student's $t$ test.

\section{Figure 7}

TWEAK pathway blockade in DDC-fed mice inhibits the proliferation of A6-expressing oval cells. DDC-fed mice were dosed with either hamster anti-TWEAK mAb, hamster control mAb, or PBS and sacrificed 7 days later. Frozen liver sections were stained for $A 6$ expression. The amount of positive staining was then quantitated using MetaMorph software. (A) Mouse fed regular diet with no DDC. (B) DDC-fed mouse. (C) Control Ig-treated DDC-fed mouse. (D) Hamster anti-TWEAK mAb-treated DDC-fed mouse. Magnification, $\times 200$. (E) Quantitation of A6 staining using MetaMorph software. ${ }^{*} P<0.05$ by 2 -tailed Student's $t$ test.

and cytokines that play a role in both oval cell and hepatocyte growth, TWEAK appears to have a selective effect on oval cells. The ability to block oval cell expansion in the DDC model by inhibiting the TWEAK pathway demonstrates that this inducible pathway is an important stimulator of hepatic stem cell responses during liver injury. Moreover, since TWEAK binds to many progenitor cells of the mesenchymal lineage, including human muscle satellite cells, preadipocytes, and chondrocytes (T. Zheng, unpublished observations), it is possible that TWEAK may be a regulator of organ progenitor cells in multiple organ systems. Its potential role in chronic human liver diseases in which progenitor cell activation is featured is suggested by the increased expression of Fn14 on the abundant duct-like structures present in alcoholic cirrhosis and viral hepatitis.

\section{Methods}

Generation of TWEAK-Tg mice. The murine transgenic construct was generated so that it contained an apoE enhancer-human AAT promoter, the fulllength murine TWEAK coding sequence (aa 1-249), and the SV40 polyA addition site. The plasmid containing the enhancer/promoter region was a generous gift from K.P. Ponder (Washington University School of Medicine, St. Louis, Missouri, USA). An EcoRV + BgIII + ScaI fragment was used for microinjection. The full-length murine TWEAK sequence is recorded in GenBank (accession no. AF030100). Full-length TWEAK-Tg C57BL/6 ×

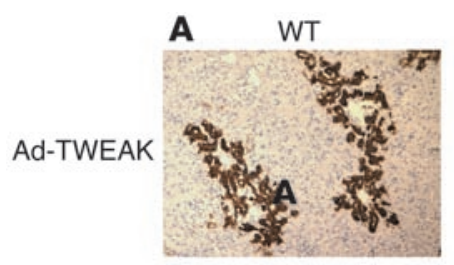

C

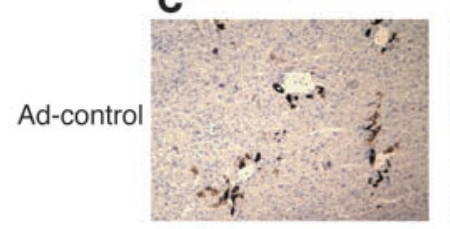

E

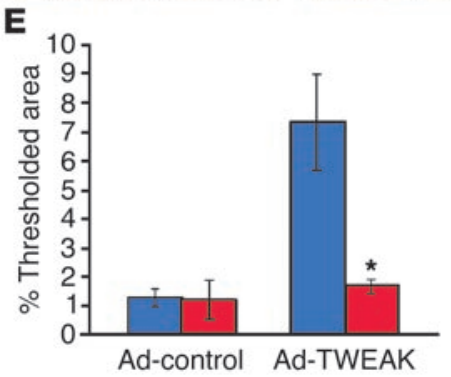

B Fn14-KO

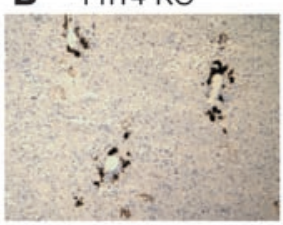

D

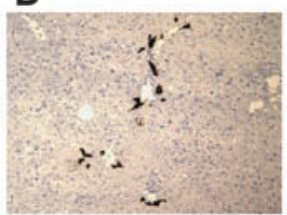

$\square W T$

$\square$ Fn14-KO 


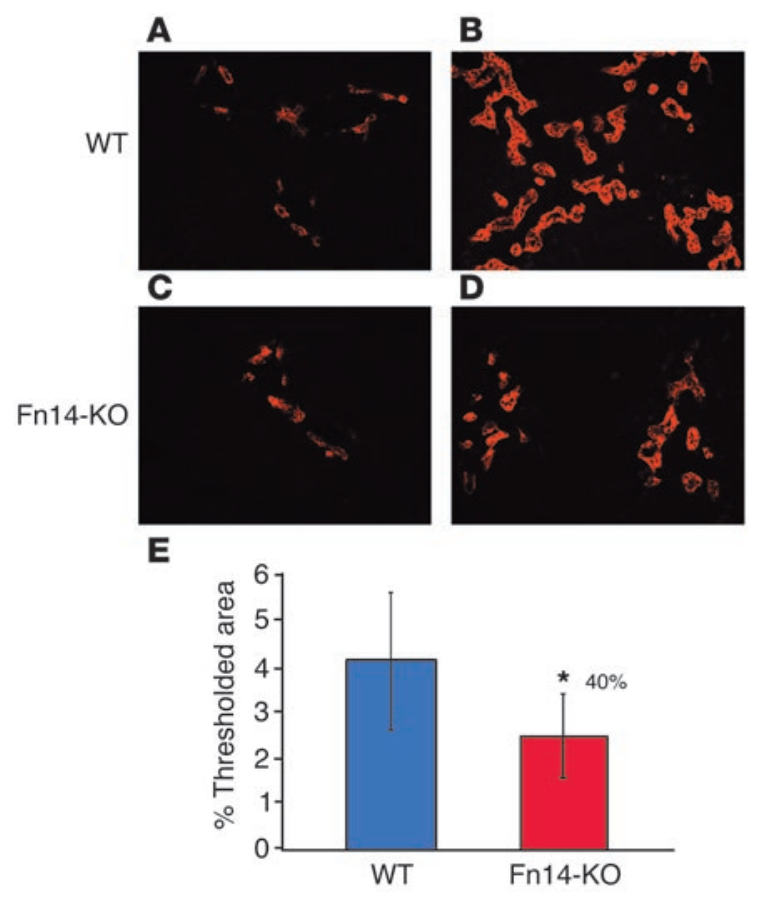

DBA/2 F1 mice were generated by M. Baetscher (Oregon Health Sciences University) using standard procedures. Mice were genotyped by PCR of genomic tail DNA for a 246-bp transgene-specific product. TWEAK-Tg mice were maintained by backcrossing to the C $57 \mathrm{BL} / 6$ strain.

Generation of Fn14-KO mice. Fn14-KO mice were generated using a standard approach. Briefly, a 10-kb Kpn1 genomic DNA fragment containing the full murine Fn14 gene was isolated, and a targeting vector was designed to delete the first 2 exons, which contained the entire extracellular ligand-binding domain of Fn14. The target vector was transfected into the J1 129 ES cell line and selected with G418. ES cell clones were screened for homologous recombination using Southern blot, and the correct clones were injected into C57BL/ 6 blastocysts to generate chimeras. Mice heterozygous for targeted Fn14 alleles were obtained through further breeding and identified using Southern blot or PCR. The null mutation was confirmed by both Northern blot and RT-PCR (data not shown). Mice were bred to homozygosity on the 129 background. The Fn 14 mutation was backcrossed 5 times onto the $\mathrm{C} 57 \mathrm{BL} / 6$ background, and wild-type and $\mathrm{KO}$ mice were intercrossed to generate cohorts for experiments.

TWEAK ELISA. Circulating TWEAK was detected in mouse serum by solid-phase ELISA using anti-TWEAK mAbs that have been previously described $(49,50)$. The BC.B10 mAb was coated onto 96-well microtiter plates and kept overnight at $4{ }^{\circ} \mathrm{C}$. Plates were then washed and blocked with PBS containing $1 \%$ BSA, after which serum or the murine version of the previously described recombinant soluble TWEAK (49) standard dilutions were added and incubated for 1 hour at room temperature. Plates were washed and TWEAK detected by a 1:1 premixture of biotinylated anti-TWEAK mAb AB.D3 and streptavidin-alkaline phosphatase (Jackson ImmunoResearch Laboratories Inc.) incubated for 1 hour at room temperature followed by the addition of 4-nitrophenylphosphat substrate (PNPP; Boehringer Mannheim).

Adenovirus preparations. The first generation adenoviral vector (E1, E3 deleted, serotype 5) expressing soluble murine TWEAK was created via standard methods in 293 cells $(51,52)$. Purification was done by double-cesium chloride density equilibrium gradient centrifugation and

\section{Figure 9}

DDC-fed Fn14-KO mice have reduced proliferation of A6-expressing oval cells. Fn14-KO mice or age-matched control littermates were fed with the DDC diet or with regular chow. Mice were sacrificed 14 days later, and frozen liver sections were stained for A6 expression, which was then quantified using MetaMorph software. Wild-type mice fed $(\mathbf{A})$ regular diet or (B) DDC-containing diet. Fn14-KO mice fed (C) regular diet or (D) DDC-containing diet. Magnification, $\times 200$. (E) Quantitation of A6 staining using MetaMorph software. ${ }^{*} P<0.02$ by 2 -tailed Student's $t$ test.

the vector stored at $-80^{\circ} \mathrm{C}(10 \mathrm{mM}$ TrisHCl, $1 \mathrm{mM} \mathrm{MgCl} 2,10 \%$ glycerol $\mathrm{vol} / \mathrm{vol}, \mathrm{pH}$ 8.0). Expression of murine TWEAK from this vector was verified using an ELISA assay both in vitro with A549 cells (ATCC) and in vivo with mice given the vector intravenously. A comparable adenoviral construct expressing GFP was used as a control.

Adenoviral delivery studies. C57BL/ 6 female mice of $6-8$ weeks purchased from Taconic Farms were used in the adenoviral delivery experiments. In some of the studies, Fn14-KO females or age-matched littermates of 8-10 weeks were used.

A total adenoviral vector dose of $10^{11}$ viral particles was used in both control and experimental mice in order to insure a good transduction efficiency. Thus, control mice were dosed with $10^{11}$ control particles (Adcontrol), while experimental mice were dosed with a mixture of $0.05 \times 10^{11}$ TWEAK-expressing particles (Ad-TWEAK) and $0.95 \times 10^{11} \mathrm{Ad}$-control particles, thus maintaining a constant total particle number. Mice were sacrificed at the time points indicated; liver samples were harvested and fixed in $4 \%$ paraformaldehyde or frozen in OCT for further analysis. All mouse studies were approved by the Institutional Animal Care and Use Committees of Biogen Idec Inc. and UCSF.

DDC-feeding protocol. Adult male C57BL/6 (25-35 g) purchased from Charles River Laboratories were used in this model. In some studies, 10to 12-week-old Fn14-KO males or age-matched control littermates were used. Mice were fed a diet containing DDC (Sigma-Aldrich). For this purpose, regular standard chow (Basal Diet 5755) was mixed with $0.1 \%$ DDC (TestDiet Inc.). For pharmacological blocking experiments, the blocking anti-TWEAK mAb AA.DG7 $(150 \mathrm{mg})$ or the same amount of a hamster IgG isotype control were injected i.p. 24 hours before the mice started the DDC-supplemented diet. Mice were dosed twice a week thereafter at 3- to

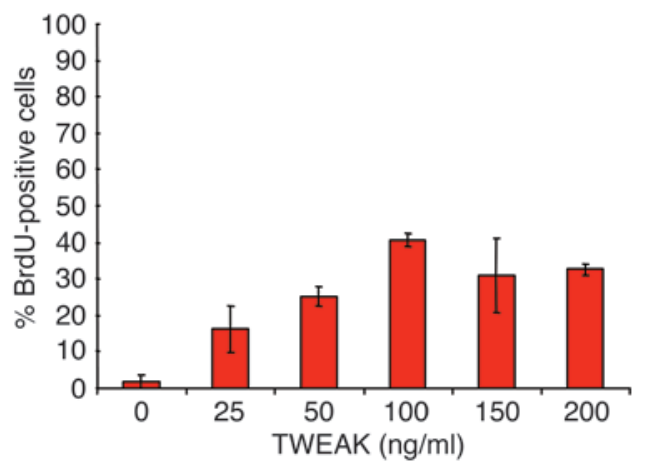

\section{Figure 10}

TWEAK induces proliferation of NRC-1 cells. NRC-1 cells were cultured with a range of TWEAK concentrations in serum-free medium for 48 hours. Then TWEAK-containing medium was renewed, with the addition of BrdU, and incubated a further 24 hours. Positive cells and total cells were counted in 10 randomly chosen fields. Results are expressed as percentage of BrdU-positive cells. Graph shows the combined results of 3 independent experiments. 


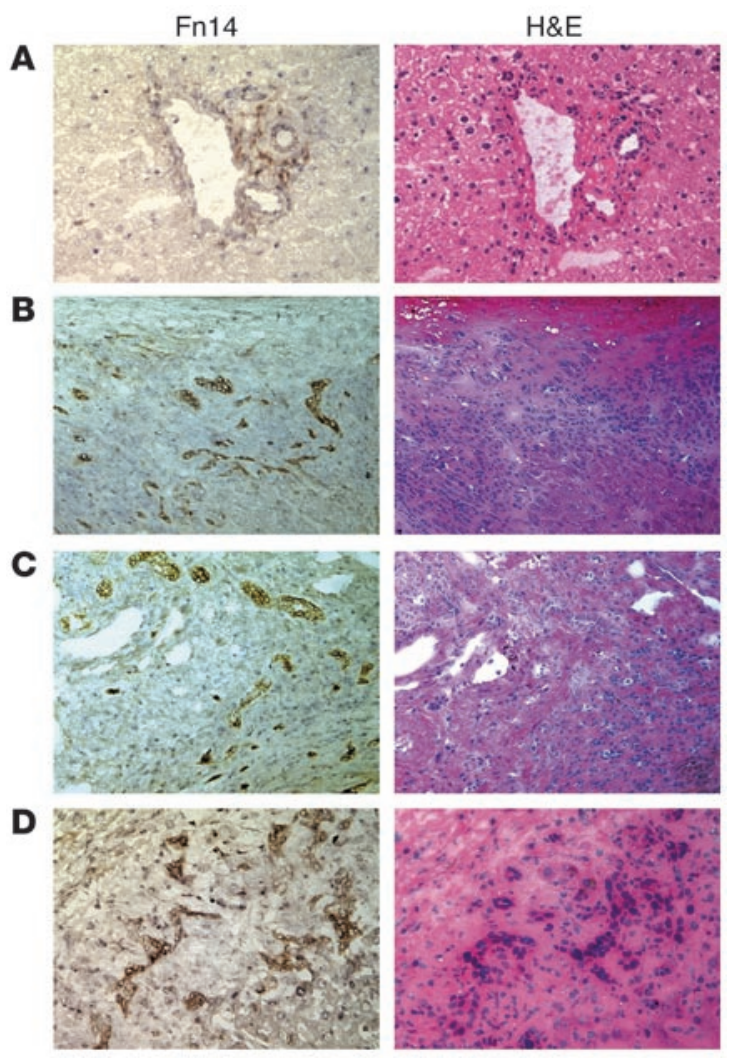

Figure 11

Fn14 expression in human liver. Serial frozen human liver sections were stained with either anti-Fn14 mAb or H\&E. (A) Normal liver. (B) NASH cirrhosis. (C) Alcoholic cirrhosis (end stage). (D) HCV with HCC. Magnification, $\times 210$.

4-day intervals. The blocking anti-TWEAK mAb used has subnanomolar affinity for TWEAK by Biacore analysis and completely inhibits soluble TWEAK binding to Fn14-expressing cells and soluble TWEAK-induced IL-8 production by tumor cells $(16,49)$. Mice were sacrificed at the indicated time points, liver and body weights were recorded, and pieces of liver were frozen in OCT or fixed in $4 \%$ paraformaldehyde.

Serum chemistry. Sera were analyzed for ALT and aspartate aminotransaminase (AST) using an Olympus AU400e Chemistry Immuno Analyzer.

Histopathological analysis. Livers were either frozen in OCT compound (Fisher Scientific) or fixed in 10\% neutral buffered formalin, paraffinembedded, and H\&E stained. Proliferating cells were detected by proliferating cell nuclear antigen (PCNA) staining of paraffin-embedded tissues using a kit (Zymed Labs). Apoptotic cells were detected using a TUNEL assay (ApopTag Peroxidase In Situ Apoptosis Detection Kit; Intergen Co.). Cells expressing PCNA or TUNEL were counted by a blinded observer at $\times 200$ magnification as indicated. For each marker, results are expressed as the total number of positive cells per 20 fields and are the average of those from 3-6 individual animals.

Immunohistochemistry. Indirect immunoperoxidase staining was performed on acetone-fixed mouse liver frozen sections using the rat primary antibodies A6 (1:60 dilution of hybridoma supernatant) and anti-CD34 $(6 \mu \mathrm{g} / \mathrm{ml}$; BD Biosciences - Pharmingen) or a hamster anti-TWEAK mAb which has previously been described $(16,49)$. Staining was detected with either biotin-conjugated rabbit anti-rat IgG (Vector) or biotin-conjugated mouse anti-hamster IgG (clones G192-1 and G94-56; BD Biosciences
- Pharmingen) and streptavidin ABC complex (DakoCytomation). For immunofluorescent staining, we costained sections with $\mathrm{A} 6$ and biotinconjugated rat anti-CD34 and detected them with the secondary antibodies Alexa Fluor 594 goat anti-rat IgG and Alexa Fluor 488 streptavidin (Invitrogen Corp.), respectively. Frozen human liver sections were from explanted livers obtained at the time of liver transplantation. Sections were stained with mouse anti-Fn14 (clone ITEM-4; eBioscience), which was detected with biotin-conjugated horse anti-mouse IgG (Vector Laboratories). Negative controls were performed using the appropriate isotype control. In some studies, expression was quantified using MetaMorph software (version 6.1; Universal Imaging Corp.).

In situ bybridization. Techniques for in situ analysis have been described in detail elsewhere $(53,54)$. Fn14 riboprobe templates were generated using specific PCR primers containing T7 polymerase promoter binding sites on either the forward or reverse primers to generate sense or antisense templates, respectively. The murine Fn 14 riboprobe template has 448 bps and is specific for nucleotides 9-456 in the coding domain (GenBank accession no. BC025860). The 226-bp TWEAK riboprobe template is specific for nucleotides $228-454$ in the murine TWEAK (TNFSF12) coding domain (GenBank accession no. AF030100). This region corresponds to coding exons $3,4,5$, and 6 of the TWEAK gene. High specific-activity probes were synthesized using an Ambion T7 MAXIscript in vitro transcription kit and ${ }^{32} \mathrm{P}$-radiolabeled UTP $(>3,000$ $\mathrm{Ci} / \mathrm{mM}$; PerkinElmer), according to the manufacturers' instructions. All probes were used in a hybridization buffer containing $30,000 \mathrm{cpm} / \mathrm{ml}$ final probe concentration. Microscopic analysis of the expression patterns was performed on a Leica DMR system modified for reflective darkfield microscopy, and pictures displayed are of a single area photographed in both darkfield and brightfield. Images were captured using a CoolSNAP RGB camera (Photometrics) and Openlab software (version 3.0.4; Improvision). Adobe Photoshop 7.0 was used to adjust the contrast for improved visualization.

Real-time PCR. RNA levels were quantified using real-time PCR on total RNA isolated from livers using the TRIzol reagent (Invitrogen Corp.) and further purified by RNeasy (QIAGEN) according to the manufacturer's instructions.

Oligonucleotide primers (20-25 bp) and TaqMan MGB probes (Applied Biosystems) for detecting murine TWEAK and Fn14 were designed from Affymetrix consensus sequences using Primer Express software, version 2.0.0 (Applied Biosystems). Murine TWEAK primer sequences were as follows: forward, 5'-CGAGCTATTGCAGCCCATTAT-3'; backward, 5'-ACCTGCTTGTGCTCCATCCT-3'. Murine Fn14 primer sequences were as follows: forward, $5^{\prime}$-CTAGTTTCCTGGTCTGGAGAAGATG3'; backward, 5'-CCСТCTCCACCAGTCTCCTCTA-3'. These sequences mapped in the extracellular domains of both TWEAK and Fn14 molecules. Therefore, TWEAK primers detected total TWEAK expression, including both endogenous and transgene expression. For the detection of TWEAK transgene, primers were purchased from Epoch Biosciences. TWEAK transgene-specific primer sequences were as follows: forward, $5^{\prime}$ TCCAGACATGATAAGATACATTGATGAG-3'; backward, 5'-GTTA*CAA ATA*AAGCAATAGCATCAC-3'. These sequences mapped at the junction between the SV40 polyA site and the murine TWEAK sequence, therefore detecting only transgene TWEAK. Probes contained a fluorescent reporter dye (FAM) covalently linked to the $5^{\prime}$ end and a minor groove binder/ nonfluorescent quencher (MGBNF) covalently linked to the $3^{\prime}$ end. The probe for total TWEAK was 5'-6FAM-AGGTTCATCCTCGGCC-3' and for Fn 14 was 5'-6FAM-AGAGAAAAGTTTACTACCCCC-3'. The probe for TWEAK transgene, purchased from Epoch Biosciences, was as follows: 5'-GACAAACCACA ACTAG-3'. Oligonucleotide standard templates were designed by the addition of $10 \mathrm{bp}$ of gene-specific sequence to the $5^{\prime}$ and 
$3^{\prime}$ ends of the amplicon. Reverse-phase HPLC-purified primers and oligonucleotide standard templates were from Biosearch Technologies, and HPLC-purified probes were from Applied Biosystems. Primers and probes for GAPDH (Applied Biosystems) served as an internal control. Assays were performed in quadruplicate in a $7900 \mathrm{HT}$ thermal cycler (Applied Biosystems) under the following conditions: 1 cycle at $50^{\circ} \mathrm{C}$ for 2 minutes (uracyl $\mathrm{N}$-deglycosylate digest); 1 cycle at $95^{\circ} \mathrm{C}$ for 10 minutes (activation of Taq thermostable polymerase); and 40 cycles at $95^{\circ} \mathrm{C}$ for 15 seconds and at $60^{\circ} \mathrm{C}$ for 60 seconds. The fluorescence emission was collected every 7 seconds for the length of the run for each reaction well. Relative transcript quantities were determined for each sample by comparison with oligonucleotide standard curve using Sequence Detection Software (version 2.2; Applied Biosystems).

Cell culture. NRC-1 cells, a cholangiocyte cell line from rat liver, were obtained from N.F. LaRusso (Mayo Clinic, Rochester, Minnesota, USA) and maintained as described. The growth medium contained $5 \%$ fetal-calf serum (55), and the substratum was of gelled collagen type I (Vitrogen-100; Angiotech BioMaterials Corp.). Under these conditions, the cells grow as a compact monolayer with the morphology of oval cells. Like oval cells in vivo, they are strongly positive for $\beta_{6}$ integrin (data not shown). Prior to study, the cells were serum-starved for 24 hours. TWEAK was then added in serum-free medium, and cultures were incubated for 48 hours. The TWEAKcontaining medium was renewed, with the addition of $\operatorname{BrdU}(50 \mu \mathrm{g} / \mathrm{ml})$, and incubated a further 24 hours. The cultures were washed, fixed, and stained with anti-BrdU using a kit from Zymed Laboratories Inc.; cells were counterstained lightly with hematoxylin. Positive cells (with brown-black nuclei) and total cells were counted in 10 randomly chosen fields.

Statistical analysis. Data are presented as mean \pm SD and were analyzed by 2 -tailed Student's $t$ test. A $P$ value less than 0.05 was considered significant.

\section{Acknowledgments}

We are indebted to Valentina Factor (National Cancer Institute, Bethesda, Maryland, USA) for her generous gift of the A6 $\mathrm{mAb}$, which was crucial for the characterization of the oval cells described in this study. We sincerely thank Norm Allaire for quantitative PCR analysis; Humphrey Gardner for his valuable histological evaluation; Thomas Crowell and Pamela Korsman for tissue processing and H\&E staining; Jorge Sanchez-Salazar for preparing the Adenovirus stock; and Wendy Mars (University of Pittsburgh, Pittsburgh, Pennsylvania, USA) for valuable discussions.

Received for publication September 28, 2004, and accepted in revised form May 31, 2005.

Address correspondence to: Aniela Jakubowski, Biogen Idec Inc., Department of Exploratory Science, 12 Cambridge Center, Cambridge, Massachusetts 02142, USA. Phone: (617) 679-6205; Fax: (617) 679-3148; E-mail: Aniela.Jakubowski@biogenidec.com.
1. Fausto, N., and Campbell, J.S. 2003. The role of hepatocytes and oval cells in liver regeneration and repopulation. Mech. Dev. 120:117-130.

2. Tan, J., et al. 2002. Immunohistochemical evidence for hepatic progenitor cells in liver diseases. Liver. 22:365-373.

3. Libbrecht, L., and Roskams, T. 2002. Hepatic progenitor cells in human liver diseases. Semin. Cell Dev. Biol. 13:389-396.

4. Roskams, T. 2003. Progenitor cell involvement in cirrhotic human liver diseases: from controversy to consensus [review]. J. Hepatol. 39:431-434.

5. Paku, S., Schnur, J., Nagy, P., and Thorgeirsson, S.S. 2001. Origin and structural evolution of the early proliferating oval cells in rat liver. Am. J. Pathol. 158:1313-1323.

6. Petersen, B.E., Zajac, V.F., and Michalopoulos, G.K. 1998. Hepatic oval cell activation in response to injury following chemically induced periportal or pericentral damage in rats. Hepatology. 27:1030-1038.

7. Sell, S. 2001. Heterogeneity and plasticity of hepatocyte lineage cells. Hepatology. 33:738-750.

8. Golding, M., et al. 1995. Oval cell differentiation into hepatocytes in the acetylaminofluorene-treated regenerating rat liver. Hepatology. 22:1243-1253.

9. Lowes, K.N., Croager, E.J., Olynyk, J.K., Abraham, L.J., and Yeoh, G.C. 2003. Oval cell-mediated liver regeneration: role of cytokines and growth factors [review]. J. Gastroenterol. Hepatol. 18:4-12.

10. Fausto, N. 2000. Liver regeneration. J. Hepatol. 32:19-31.

11. Oh, S.H., Hatch, H.M., and Petersen, B.E. 2002. Hepatic oval 'stem' cell in liver regeneration. Semin. Cell Dev. Biol. 13:405-409.

12. Nagy, P., Bisgaard, H.C., Santoni-Rugiu, E., and Thorgeirsson, S.S. 1996. In vivo infusion of growth factors enhances the mitogenic response of rat hepatic ductal (oval) cells after administration of 2-acetylaminofluorene. Hepatology. 23:71-79.

13. Fujio, K., et al. 1996. Coexpression of stem cell factor and c-kit in embryonic and adult liver. Exp. Cell Res. 224:243-250.

14. Shiota, G., et al. 2000. In vivo transfer of hepatocyte growth factor gene accelerates proliferation of hepatic oval cells in a 2-acetylaminofluorene/ partial hepatectomy model in rats. FEBS Lett.
470:325-330

15. Knight, B., et al. 2000. Impaired preneoplastic changes and liver tumor formation in tumor necrosis factor receptor type 1 knockout mice. J. Exp. Med. 192:1809-1818.

16. Chicheportiche, Y., et al. 1997. TWEAK, a new secreted ligand in the tumor necrosis factor family that weakly induces apoptosis. J. Biol. Chem. 272:32401-32410.

17. Wiley, S.R., and Winkles, J.A. 2003. TWEAK, a member of the TNF superfamily, is a multifunctional cytokine that binds the TweakR/Fn14 receptor. Cytokine Growth Factor Rev. 14:241-249.

18. Campbell, S., Michaelson, J., Burkly, L., and Putterman, C. 2004. The role of TWEAK/Fn14 in the pathogenesis of inflammation and systemic autoimmunity. Front. Biosci. 9:2273-2284.

19. Wiley, S.R., et al. 2001. A novel TNF receptor family member binds TWEAK and is implicated in angiogenesis. Immunity. 15:837-846.

20. Feng, S.L., et al. 2000. The Fn14 immediate-early response gene is induced during liver regeneration and highly expressed in both human and murine hepatocellular carcinomas. Am. J. Pathol. 156:1253-1261.

21. Meighan-Mantha, R.L., et al. 1999. The mitogeninducible Fn14 gene encodes a type I transmembrane protein that modulates fibroblast adhesion and migration. J. Biol. Chem. 274:33166-33176.

22. Shen, R.F., et al. 1989. Tissue-specific regulation of human alpha 1-antitrypsin gene expression in transgenic mice. DNA. 8:101-108.

23. Engelhardt, N.V., et al. 1993. Common antigen of oval and biliary epithelial cells (A6) is a differentiation marker of epithelial and erythroid cell lineages in early development of the mouse. Differentiation. 55:19-26.

24. Factor, V.M., and Radaeva, S.A. 1993. Oval cells-hepatocytes relationships in Dipin-induced hepatocarcinogenesis in mice. Exp. Toxicol. Pathol. 45:239-244.

25. Petersen, B.E., et al. 2003. Mouse A6-positive hepatic oval cells also express several hematopoietic stem cell markers. Hepatology. 37:632-640.

26. Omori, N., et al. 1997. Partial cloning of rat CD34 cDNA and expression during stem cell-dependent liver regeneration in the adult rat. Hepatology. 26:720-727.

27. Lemaigre, F.P. 2003. Development of the biliary tract. Mech. Dev. 120:81-87.

28. Meehan, R.R., Barlow, D.P., Hill, R.E., Hogan, B.L., and Hastie, N.D. 1984. Pattern of serum protein gene expression in mouse visceral yolk sac and foetal liver. EMBO J. 3:1881-1885.

29. Nakayama, M., Kayagaki, N., Yamaguchi, N., Okumura, K., and Yagita, H. 2000. Involvement of TWEAK in interferon gamma-stimulated monocyte cytotoxicity. J. Exp. Med. 192:1373-1380.

30. Schneider, P., et al. 1999. TWEAK can induce cell death via endogenous TNF and TNF receptor 1. Eur. J. Immunol. 29:1785-1792.

31. Nakayama, M., et al. 2003. Fibroblast growth factorinducible 14 mediates multiple pathways of TWEAKinduced cell death. J. Immunol. 170:341-348.

32. Nakayama, M., et al. 2002. Multiple pathways of TWEAK-induced cell death. J. Immunol. 168:734-743.

33. Roskams, T.A., Libbrecht, L., and Desmet, V.J. 2003. Progenitor cells in diseased human liver. Semin. Liver Dis. 23:385-396.

34. Evarts, R.P., Hu, Z., Fujio, K., Marsden, E.R., and Thorgeirsson, S.S. 1993. Activation of hepatic stem cell compartment in the rat: role of transforming growth factor alpha, hepatocyte growth factor, and acidic fibroblast growth factor in early proliferation. Cell Growth Differ. 4:555-561.

35. Fujio, K., Evarts, R.P., Hu, Z., Marsden, E.R., and Thorgeirsson, S.S. 1994. Expression of stem cell factor and its receptor, c-kit, during liver regeneration from putative stem cells in adult rat. $L a b$. Invest. 70:511-516.

36. Omori, N., et al. 1996. Expression of leukemia inhibitory factor and its receptor during liver regeneration in the adult rat. Lab. Invest. 75:15-24.

37. Nagy, P., Kiss, A., Schnur, J., and Thorgeirsson, S.S. 1998. Dexamethasone inhibits the proliferation of hepatocytes and oval cells but not bile duct cells in rat liver. Hepatology. 28:423-429.

38. Cressman, D.E., et al. 1996. Liver failure and defective hepatocyte regeneration in interleukin-6-deficient mice. Science. 274:1379-1383.

39. Yamada, Y., Kirillova, I., Peschon, J.J., and Fausto, N. 1997. Initiation of liver growth by tumor necro- 
sis factor: deficient liver regeneration in mice lacking type I tumor necrosis factor receptor. Proc. Natl. Acad. Sci. U. S. A. 94:1441-1446.

40. Tee, L.B., Kirilak, Y., Huang, W.H., Morgan, R.H., and Yeoh, G.C. 1994. Differentiation of oval cells into duct-like cells in preneoplastic liver of rats placed on a choline-deficient diet supplemented with ethionine. Carcinogenesis. 15:2747-2756.

41. Sanchez, A., Factor, V.M., Schroeder, I.S., Nagy, P., and Thorgeirsson, S.S. 2004. Activation of NF-kappaB and STAT3 in rat oval cells during 2-acetylaminofluorene/partial hepatectomy-induced liver regeneration. Hepatology. 39:376-385.

42. Hixson, D.C., Brown, J., McBride, A.C., and Affigne, S. 2000. Differentiation status of rat ductal cells and ethionine-induced hepatic carcinomas defined with surface-reactive monoclonal antibodies. Exp. Mol. Pathol. 68:152-169.

43. Lowes, K.N., Brennan, B.A., Yeoh, G.C., and Olynyk, J.K. 1999. Oval cell numbers in human chronic liver diseases are directly related to disease severity. Am. J. Pathol. 154:537-541.
44. Polek, T.C., Talpaz, M., Darnay, B.G., and Spivak-Kroizman, T. 2003. TWEAK mediates signal transduction and differentiation of RAW264.7 cells in the absence of Fn14/TweakR. Evidence for a second TWEAK receptor. J. Biol. Chem. 278:32317-32323.

45. Sell, S., and Dunsford, H.A. 1989. Evidence for the stem cell origin of hepatocellular carcinoma and cholangiocarcinoma. Am. J. Pathol. 134:1347-1363.

46. Dumble, M.L., Croager, E.J., Yeoh, G.C., and Quail, E.A. 2002. Generation and characterization of p53 null transformed hepatic progenitor cells: oval cells give rise to hepatocellular carcinoma. Carcinogenesis. 23:435-445.

47. Tsukuma, H., et al. 1993. Risk factors for hepatocellular carcinoma among patients with chronic liver disease. N. Engl. J. Med. 328:1797-1801.

48. Prior, P. 1988. Long-term cancer risk in alcoholism. Alcohol Alcohol. 23:163-171.

49. Jakubowski, A., et al. 2002. Dual role for TWEAK in angiogenic regulation. J. Cell Sci. 115:267-274.

50. Chicheportiche, Y., et al. 2002. Proinflammatory activity of TWEAK on human dermal fibroblasts and synoviocytes: blocking and enhancing effects of anti-TWEAK monoclonal antibodies. Arthritis Res. 4:126-133.

51. Ng, P., Parks, R.J., Cummings, D.T., Evelegh, C.M., and Graham, F.L. 2000. An enhanced system for construction of adenoviral vectors by the two-plasmid rescue method. Hum. Gene Ther. 11:693-699.

52. Ng, P., et al. 1999. A high-efficiency Cre/loxP-based system for construction of adenoviral vectors. Hum. Gene Ther. 10:2667-2672.

53. Sassoon, D.A., Garner, I., and Buckingham, M. 1988. Transcripts of alpha-cardiac and alpha-skeletal actins are early markers for myogenesis in the mouse embryo. Development. 104:155-164.

54. Sassoon, D., and Rosenthal, N. 1993. Detection of messenger RNA by in situ hybridization. Methods Enzymol. 225:384-404.

55. Vroman, B., and LaRusso, N.F. 1996. Development and characterization of polarized primary cultures of rat intrahepatic bile duct epithelial cells. Lab. Invest. 74:303-313. 